\title{
Measurement of Skull Size on Computed Tomography Images for Developing a Bone Conduction Headset Suitable for the Korean Standard Head Size
}

\author{
Cheol Hyo Ku${ }^{1}$, Soo Won Kim², Ji Young Kim², Seung Won Paik', \\ Hui Joon Yang ${ }^{1}$, Ji Hyeon Lee ${ }^{1,3}$, and Young Joon Seo ${ }^{1,3}$ \\ Departments of ${ }^{1}$ Otorhinolaryngology and ${ }^{2}$ Medicine, and ${ }^{3}$ Research Institute of Hearing Enhancement, \\ Yonsei University Wonju College of Medicine, Wonju, Korea
}

Received July 16, 2019

Revised August 10, 2019

Accepted August 10, 2019

Address for correspondence

Young Joon Seo, MD, PhD

Department of Otorhinolaryngology,

Yonsei University

Wonju College of Medicine,

20 Ilsan-ro, Wonju 26426, Korea

Tel $+82-33-741-0644$

Fax $+82-33-732-8287$

E-mail okas2000@yonsei.ac.kr
Background and Objectives: We aimed to measure the head dimensions on computed tomography (CT) images, to compare them to directly measured head dimensions, and to predict a new parameter of bone thickness for aiding bone conduction implant (BCl) placement. Subjects and Methods: We reviewed the facial and mandibular bone CT images of 406 patients. Their head sizes were analyzed using five parameters included in the 6th Size Korea project, and they were divided into age groups (ranging from the 10s to the 80s). We compared the head length, head width, sagittal arc, bitragion arc, and head circumference in the CT and Size Korea groups. We also added the parameter bone thickness for aiding $\mathrm{BCl}$ placement. Results: All the head size parameters measured using CT were significantly smaller than those measured directly, with head length showing the smallest difference at $7.85 \mathrm{~mm}$. The differences in the other four parameters between the two groups according to patient age were not statistically significantly different. Bone thickness had the highest value of $4.89 \pm 0.93$ $\mathrm{mm}$ in the $70 \mathrm{~s}$ and the lowest value of $4.10 \pm 0.99 \mathrm{~mm}$ in the $10 \mathrm{~s}$. Bone thickness also significantly correlated with head width $(p=0.038)$. Conclusions: Our findings suggested that the CT and direct measurements yielded consistent data. Moreover, CT enabled the measurement of bone sizes, including bone thickness, that are impossible to measure directly. CT measurements may complement direct measurements in the Size Korea data when used for developing bone conduction hearing devices (BCls and headsets) for the Korean population.

J Audiol Otol 2020;24(1):17-23

KEY WORDS: Bone conduction implant · Headset · Size Korea · Computed tomography · Temporal bone.

\section{Introduction}

Bone conduction hearing aids can transmit sound vibrations directly to the inner ear through the skull, thereby bypassing any conductive impairment in the external or middle ear [1]. Patients with recurrent otitis media or otitis exacerbation find it difficult to wear conventional air-conduction hearing aids. Moreover, compared to bone conduction implants (BCIs), con-

This is an Open Access article distributed under the terms of the Creative Commons Attribution Non-Commercial License (https://creativecommons.org/licenses/by-nc/4.0/) which permits unrestricted non-commercial use, distribution, and reproduction in any medium, provided the original work is properly cited. ventional hearing aids have disadvantages such as the damping of sound vibrations by the hearing aid and the inconvenience due to irritation of the attachment area. BCIs are good alternative options for these patients [2,3]. Since the successful placement of the first implant was reported in 1977, more than 10,000 patients worldwide have received BCIs [1]. In addition, conventional bone conduction hearing aids, such as the soft Headband, are a gold standard for preoperative evaluation in young patients who cannot undergo implantation and are used as a good method for hearing rehabilitation in patients refusing surgery $[4,5]$.

To maximize the gain of sound conduction, the bone con- 
duction vibrator should be kept under a constant pressure (approximately $2-5 \mathrm{~N}$ ) on the head [6]. The shape of the headset body determines the degree of contact and pressure between the head and the device, and it plays an important role in the efficacy of sound transmission. Although many bone conduction hearing aids have been developed and used, most of them are foreign products designed for different populations; hence, they are not suitable for the heads of Korean adults. Studies have also shown that, compared to the Caucasians, Asians have heads that are more round, with flatter foreheads and backs [7]. Therefore, products designed using different population data may not be suitable for Koreans.

The human body measurement research project for Koreans is currently in progress and in its 7th stage. This project has yielded useful data for designing products and devices in various medical fields. However, among the Korean human body measurements, the head size measurements may present some limitations. The head size was mainly measured in people aged less than 69 years old, with relatively less data being collected from the elderly. Moreover, the project determined the size of the head by using five criteria, which did not account for the actual wearing position of the bone conduction headset. Furthermore, the thickness of the temporal bone to which the BCI was applied to could not been determined.

Human anthropometric measurements determined using computed tomography (CT) images have been reported in various studies [8]. In particular, CT enables the measurement of any diameter of the skull bones that cannot be measured directly. In this study, we measured the head dimensions on CT images and compared them to direct measurements of the human head. We aimed to supplement existing head size data with data from our analyzed parameters on CT images and to predict a new parameter of bone thickness for aiding BCI placement.

\section{Subjects and Methods}

\section{Patients}

This study was performed at Wonju Severance Christian Hospital and was approved by the Institutional Review Board (CR318037). Facial and mandibular bone CT images of patients acquired between January 1, 2017 and July 1, 2018 were reviewed. The patients' age at the time of acquiring the $\mathrm{CT}$ images had to range from 10 to 90 years old, because we aimed to classify the head sizes according to the ages ranging from the 10 s to the 80 s.

The exclusion criteria were as follows: 1) images that did not include a vertex and could not be used to measure the skull size; 2) patients with a past history of conditions affecting skull size measurement, e.g., skull fracture or brain surgery; and 3) patients without clinical data such as weight and height. Finally, we enrolled 406 patients in this study. The age of the patients ranged from 10 to 88 years, and their mean age was $48.58 \pm$ 22.24 years. Of the 406 patients, 221 (54.4\%) were males and $185(45.6 \%)$ were females. Patients were divided into groups according to age and included 42 to 63 people in each group $(10-19,20-29,30-39,40-49,50-59,60-69,70-79$, and $80-89)$.

The 6th Size Korea project included measurements from 14,016 people (7,532 males and 6,484 females) aged 7 to 69 years old, whose head size parameters were directly measured. Using propensity score matching, considering the height and weight of the experimental group, we were able to select 1,218 matched individuals, i.e., three times the number of patients with CT data.

\section{Measurements in the Size Korea project}

The 6th Size Korea project included five parameters related to skull size, namely, head length, head width, sagittal arc, bitragion arc, and head circumference. The definitions of the measurement parameters and the measurement reference points are summarized in Table 1.

1) Head length: The distance from the glabella to the occiput

2) Head width: The horizontal distance between the right and left euryon

3) Sagittal arc: The length from the glabella to the inion

4) Bitragion arc: The length from the right tragion, passing the vertex, to the left tragion

5) Head circumference: The perimeter through the glabella and the occiput

Two experimenters were trained to become accustomed with the measurement tools and procedures. The features were initially identified as skeletal landmarks on the face. Measurements were obtained to the nearest millimeter and were recorded in centimeters. Normally, the investigators worked in a private room to provide the subject the most preferable environment. All subjects were provided a non-disclosure agreement to preserve their names.

\section{Measurements on the CT images}

Facial and mandibular bone CT was performed using GE Light Speed Pro 16 (GE, Milwaukee, WI, USA) and Philips Brilliance 64 (Philips, Cleveland, OH, USA) (thickness, $2.5 \mathrm{~mm}$ ). The patients underwent axial topography parallel orbito-meatal line scanning in the normal supine position. Coronal and sagittal images were reconstructed perpendicular to the orbital plane and perpendicular to the axial plane, respectively. 
The five skull-size-related parameters were measured on facial and mandibular bone CT images. We additionally measured temporal bone thickness, which could not be directly measured. Temporal bone thickness was measured at a point $3 \mathrm{~cm}$ posterior and $2 \mathrm{~cm}$ superior to the posterior wall of the external auditory canal (EAC), which is the typical location of BCI placement.

These measurements were performed by two separate researchers. The lengths of straight-line parameters (head length, head width, and thickness) were measured using the Centricity software (Centricity; GE Medical Systems, Milwaukee, WI, USA), and the lengths of curved parameters, such as head circumference, bitragion arc, and sagittal arc, were measured using Image J (National Institutes of Health, Bethesda, MD, USA). The measurements were based on the bone rather than the soft tissue.

Table 1 lists the definitions and reference points of the parameters used in the CT image measurements. Fig. 1 provides an example of parameter measurements on $\mathrm{CT}$ images and di- rect measurements.

\section{Statistical analysis}

Statistical analysis was performed using IBM SPSS Statistics for Windows, Version 23.0 (IBM Corp., Armonk, NY, USA). Propensity score matching was performed, and a 1:3 ratio matching (CT group:Size Korea group) was done considering the height, weight, and body mass index of the CT group. A paired t-test was performed to analyze the two groups. A $p$-value less than 0.05 was considered to indicate a significant difference.

\section{Results}

Comparison of measurements of head size between the Size Korea project and the CT images

The measured head size parameter values are provided in detail in Table 2. The head size parameter values measured on CT images were significantly smaller than those measured di-

Table 1. Definition of head size parameters in the Size Korea project and in CT images

\begin{tabular}{|c|c|c|}
\hline Parameter & Definition in the Size Korea project & Definition in the CT images \\
\hline Head length & $\begin{array}{l}\text { The distance from the glabella to } \\
\text { the occiput }\end{array}$ & $\begin{array}{l}\text { The distance from the eyebrow to the protruding point on the } \\
\text { posterior head along the highest plane of the nose }\end{array}$ \\
\hline Head width & $\begin{array}{l}\text { The horizontal distance between the right } \\
\text { and left euryon }\end{array}$ & $\begin{array}{l}\text { The horizontal distance from the plane where the point near } \\
\text { the left head protrudes the most }\end{array}$ \\
\hline Sagittal arc & The length from the glabella to the inion & $\begin{array}{l}\text { The distance from the eye to the point at the back of the } \\
\text { head through the nose along the highest plane of the nose }\end{array}$ \\
\hline Bitragion arc & $\begin{array}{l}\text { The length from the right tragion, passing } \\
\text { the vertex, to the left tragion }\end{array}$ & $\begin{array}{l}\text { Length from the plane of the ear canal to the plane of the } \\
\text { opposite ear canal ( } 2-3 \text { planes behind the ear ball) }\end{array}$ \\
\hline Head circumference & $\begin{array}{l}\text { The perimeter through the glabella } \\
\text { and the occiput }\end{array}$ & $\begin{array}{l}\text { Head circumference from the plane where the head } \\
\text { protrudes the most }\end{array}$ \\
\hline Bone thickness & - & $\begin{array}{c}\text { The thickness of the temporal bone measured at a point } 3 \mathrm{~cm} \\
\text { posterior and } 2 \mathrm{~cm} \text { superior to the posterior wall of the EAC }\end{array}$ \\
\hline CT: computed tomog & aphy, EAC: external auditory canal & \\
\hline
\end{tabular}

Fig. 1. Illustration of the measurement of head length (A), head width (B), sagittal arc (C), bitragion arc (D), and head circumference (E). We used the Image $\mathrm{J}$ program and Centricity web program to measure the lengths on the computed tomography (CT) images. However, the CT measurements were based on the bone rather than the soft tissue. 
rectly on the human body. The average difference was the greatest for head circumference at $49.5 \mathrm{~mm}(552.45 \pm 13.17 \mathrm{~mm}$ in the Size Korea measurement and 502.80 $\pm 24.26 \mathrm{~mm}$ in the CT measurement; $p<0.001$ ), and it was the smallest for head length at $7.85 \mathrm{~mm}(178.42 \pm 6.21 \mathrm{~mm}$ in the Size Korea measurement and $170.86 \pm 8.30 \mathrm{~mm}$ in the CT measurement; $p<0.001)$.

\section{Changes in head size parameters in the CT images according to age}

The differences in four parameters (except for head length) between the two groups according to the age groups were not statistically significantly different. The changes in head size parameters measured on CT images according to the age groups are presented in Table 3. The relationship between the Size Korea project's directly measured data and CT data according to the age groups is shown in Fig. 2. This implied that all measurements, excluding the soft tissues, performed in the Size Korea project were consistent with the measurements we performed on CT images. The head lengths measured on CT images in young patients (ages in the 10s and the 20s) were considerably larger than those in the Size Korea data. This could be because in the Size Korea data, the measurements of human body size showed a steep increase until the 20 s, showing the highest values, but gradually decreased thereafter from the $30 \mathrm{~s}$.

\section{Prediction of temporal bone thickness in Koreans according to age groups}

We measured temporal bone thickness for aiding BCI placement by using CT data, because it cannot be directly measured using the Size Korea data. Bone thickness is an important factor that determines the benefit of sound gain as well as the surgical indication for BCI placement. The highest value was $4.89 \pm 0.93 \mathrm{~mm}$ in the $70 \mathrm{~s}$ and the lowest value was $4.10 \pm 0.99$ $\mathrm{mm}$ in the $10 \mathrm{~s}$ (Table 3). Correlation analysis (Fig. 3) was performed between the five head size parameters and temporal bone thickness, and only head width showed a significant correlation $\left(\mathrm{r}^{2}=0.204, p=0.038\right)$.

\section{Discussion}

Bone conduction hearing aids transmit sound vibrations through the skin to the skull bone, and further to the cochlea in the inner ear, bypassing any conductive impairment in the external or middle ear $[9,10]$. Therefore, bone conduction hearing aids or headsets are an option for treating deafness. Recently, non-invasive bone conduction hearing aids, such as the Baha SoundArc (Cochlear, Sydney, Australia) and Adhear (MED-EL, Innsbruck, Austria), have been designed to be placed over the ears and be worn behind the head $[2,11]$. SoundArc demonstrated equal performance to the Softband and superior performance to the Headband and Testband [12]. The performance of nonsurgical bone conduction solutions relies mainly on two factors: sound transfer efficiency and minimizing feedback. To solve these problems, bone conduction devices should be attached to the mastoid area with appropriate pressure to transmit the sound, and the contact force should be about 2-5 N [6]. Bone conduction devices should be fixed without any gaps at the attachment site; otherwise, they will have a negative impact on sound transmis-

Table 2. Comparison of head size parameter values between the Size Korea project and CT images

\begin{tabular}{lccc}
\hline \multicolumn{1}{c}{ Measure } & Values in the Size Korea project $(n=1,218)$ & The values in the CT images $(n=406)$ & $p$-value \\
\hline Head length & $178.42 \pm 6.21$ & $170.86 \pm 8.30$ & $<0.001$ \\
Head width & $153.20 \pm 5.89$ & $144.56 \pm 6.66$ & $<.001$ \\
Sagittal arc & $329.10 \pm 17.48$ & $297.84 \pm 18.06$ & $<0.001$ \\
Bitragion arc & $363.53 \pm 12.38$ & $319.15 \pm 23.39$ & $<0.001$ \\
Head circumference & $552.45 \pm 13.17$ & $502.80 \pm 24.26$ & $<0.001$ \\
\hline
\end{tabular}

Values are presented as mean \pm standard deviation unless otherwise indicated. CT: computed tomography

Table 3. Changes in head size parameter values in the CT images according to patient age

\begin{tabular}{|c|c|c|c|c|c|c|}
\hline Age & Head length & Head width & Sagittal arc & Bitragion arc & Head circumference & Bone thickness \\
\hline $10 \mathrm{~s}$ & $179.50 \pm 8.43$ & $146.53 \pm 5.58$ & $298.21 \pm 20.09$ & $335.39 \pm 17.44$ & $509.35 \pm 23.40$ & $4.10 \pm 0.99$ \\
\hline $20 \mathrm{~s}$ & $174.81 \pm 8.28$ & $146.47 \pm 6.44$ & $302.14 \pm 19.44$ & $328.81 \pm 20.13$ & $510.52 \pm 25.00$ & $4.13 \pm 0.88$ \\
\hline $30 s$ & $170.99 \pm 7.63$ & $145.38 \pm 7.54$ & $293.36 \pm 14.34$ & $321.30 \pm 22.13$ & $507.99 \pm 17.43$ & $4.14 \pm 1.10$ \\
\hline $40 \mathrm{~s}$ & $170.79 \pm 7.23$ & $142.67 \pm 6.84$ & $296.77 \pm 17.65$ & $315.93 \pm 19.95$ & $502.73 \pm 17.61$ & $4.58 \pm 0.78$ \\
\hline $50 \mathrm{~s}$ & $165.36 \pm 6.64$ & $140.76 \pm 6.73$ & $294.60 \pm 15.71$ & $315.71 \pm 37.09$ & $486.80 \pm 28.98$ & $4.24 \pm 1.28$ \\
\hline $60 \mathrm{~s}$ & $168.73 \pm 9.09$ & $144.40 \pm 4.82$ & $295.19 \pm 22.06$ & $307.41 \pm 17.93$ & $497.16 \pm 18.42$ & $4.21 \pm 0.95$ \\
\hline $70 \mathrm{~s}$ & $170.68 \pm 8.05$ & $141.25 \pm 6.39$ & $304.24 \pm 13.88$ & $312.02 \pm 14.08$ & $504.15 \pm 14.87$ & $4.89 \pm 0.93$ \\
\hline $80 s$ & $168.68 \pm 7.39$ & $144.56 \pm 6.66$ & $298.70 \pm 17.13$ & $303.37 \pm 12.95$ & $495.35 \pm 32.56$ & $4.74 \pm 0.97$ \\
\hline
\end{tabular}

Values are presented as mean \pm standard deviation. СТ: computed tomography 

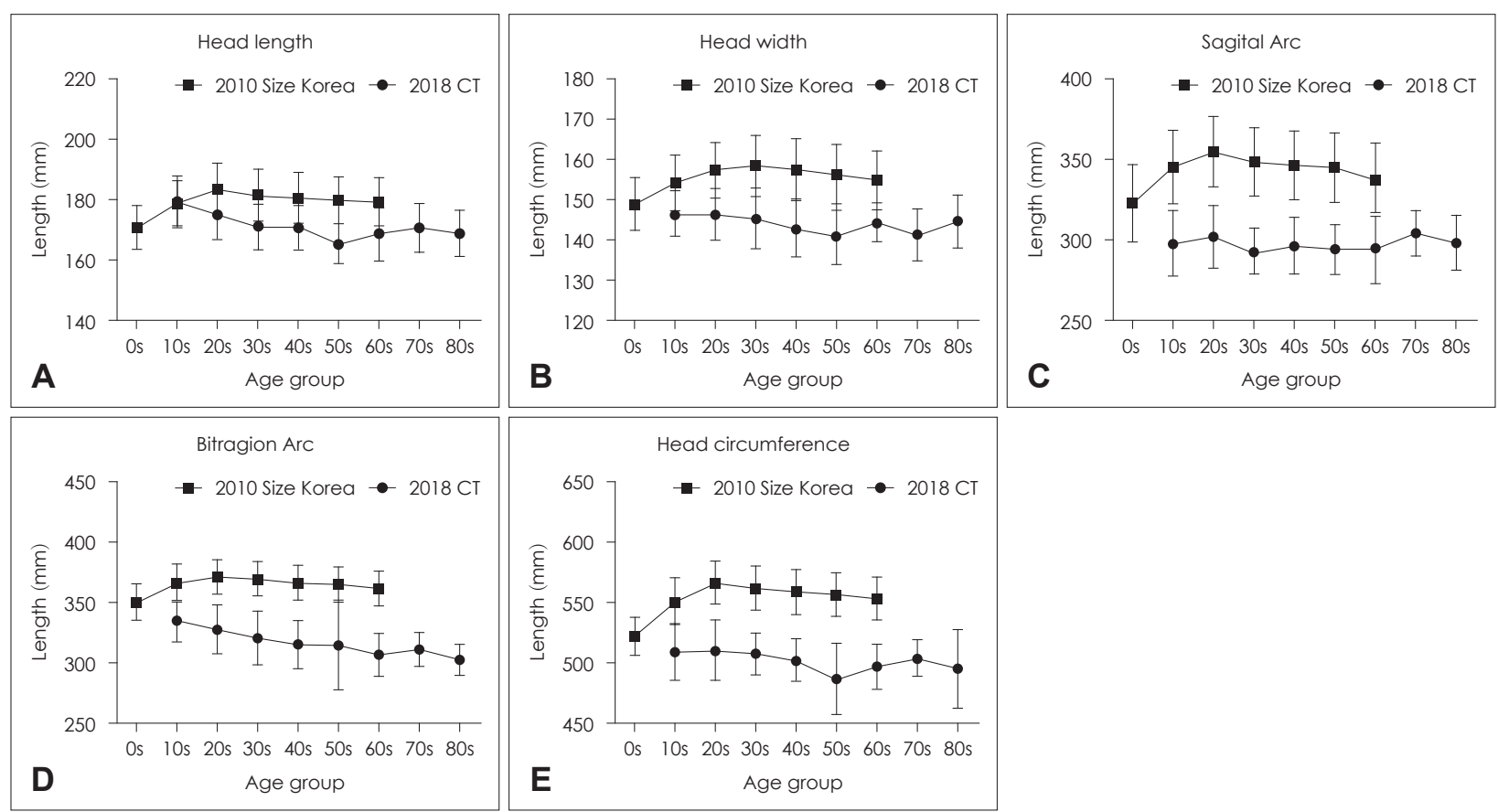

Fig. 2. Changes in head size parameters (A-E) according to ages from 10 years to 80 years. CT: computed tomography.

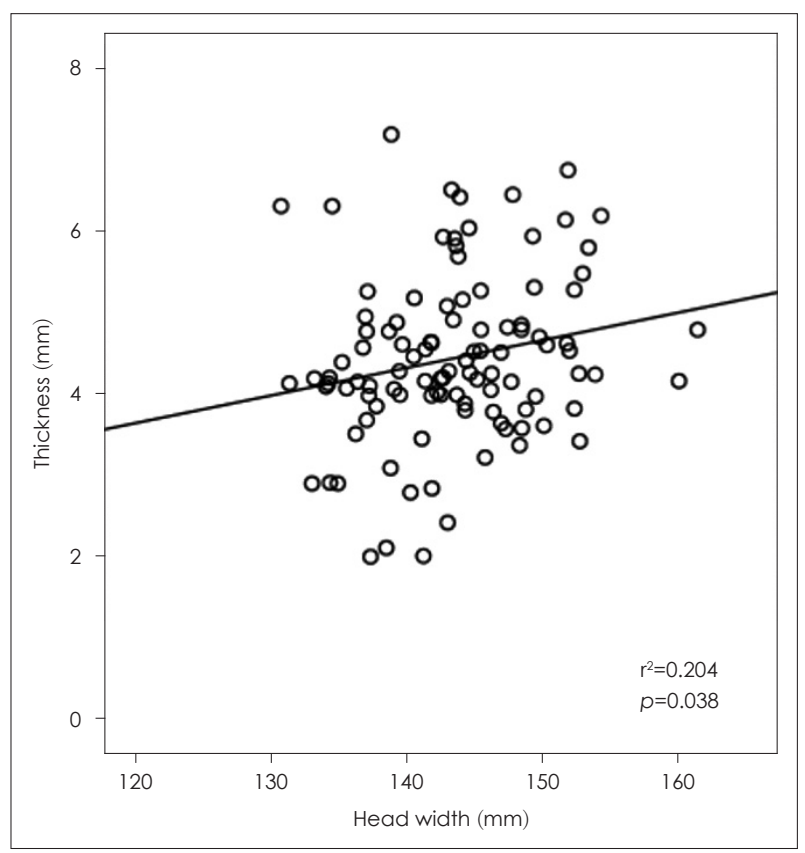

Fig. 3. The correlation between bone thickness $(\mathrm{mm})$ and head width $(\mathrm{mm})$. Bone thickness increased with an increase in head width.

sion. Head size is an important point to consider when developing devices such as bone conduction headsets and hearing aids. Most bone conduction devices are designed using reference standards obtained from foreign patient populations. Therefore, such devices may not have the maximum efficiency when used in Korean patients with different head sizes. Therefore, to develop a product suitable for Koreans, the establishment of a reference standard for the Korean head size is pertinent. Although the Size Korea project provides head size data, these are not widely used because they have limited applicability when developing medical devices. Temporal bone thickness data should be obtained to plan the position of BCIs and accomplish a successful surgery.

Anthropometric data are important factors in the design of medical devices. However, in reality, head and face shapes differ between individuals of different nationalities. Therefore, it is imperative to consider these differences in areas of object, system, and environmental design. Few studies have calculated the Korean head size. A single photon emission CT study on children suggested a series of Korean computational head phantoms with detailed cranial substructures [13]. Lee, et al. [5] showed that the face and head sizes of Korean male and female individuals have more statistically significant morphological differences than do those of Japanese individuals. Since 2003, the Korean anthropometric data survey project named Size Korea has provided Korean body size reference data, including head size data. Knowledge of the human head form is essential in a variety of fields like design and medicine $[7,14]$. However, the Size Korea project included only five head size parameters, which limited their applicability when designing medical devices, especially BCIs.

We thought that the measurement of head size on CT images could provide additional reference data to complement the Size Korea project's anthropometric data, and hence, we compared the head size data measured on CT images to those directly 
measured for the Size Korea project. In this study, the values measured on CT images were lower than the corresponding Size Korea values. We think that the CT values were lower because 1) we measured the size of the bone rather than the soft tissue and 2) the measurement points were not exactly identical between the two measurement groups; moreover, errors may have occurred during the measurements. Although the measured CT values were smaller, the five head size parameters in both the groups showed a similar tendency when viewed on the numerical graph based on the age groups. The observation of a similar tendency according to the age groups shows the reliability of the CT measurement method.

We think that CT measurements have some strengths over direct measurements. CT enables the measurement of bone sizes that are impossible to measure directly. This would be meaningful when designing bone conduction devices, which should have a contact with appropriate pressure on the bone. In addition, we could obtain and include data from patients in their $70 \mathrm{~s}$ and $80 \mathrm{~s}$, and could determine new parameters such as temporal bone thickness without re-imaging [15]. Therefore, if sufficient CT data are available in the future, they could complement national reference data (such as the Size Korea data) when developing bone conduction devices for the Korean population.

A limitation of this study was that most of the patients with CT data were from the Chungbuk or Gangwon provinces. Therefore, these patients are not representative of the whole Korean population. However, there is no evidence showing that head size changes in different regions of Korea. Therefore, we think these data can serve as meaningful standards for the Korean population.

The position of bone conduction devices is important, because it plays a role in sound transmission. According to the literature, the proximity of a bone conduction device to the cochlea is important in improving the amplification of the signal provided to the cochlea [16]. Simultaneously, avoiding the sigmoid sinus can prevent bleeding and epidural hematoma [17]. In this study, we measured temporal bone thickness at a point $3 \mathrm{~cm}$ posterior and $2 \mathrm{~cm}$ superior to the posterior wall of the EAC, where BCIs are usually placed [18]. The mean thickness ranged from $4.10 \pm 0.99 \mathrm{~mm}$ to $4.89 \pm 0.93 \mathrm{~mm}$, showing sufficient depth for BCI placement. The correlation between the five parameters and temporal bone thickness showed that bone thickness increased only with an increase in head width $\left(\mathrm{r}^{2}=0.204, p=0.038\right)$.

In conclusion, the CT-based measurement method described here may help uncover important predictive factors through modeling, and it may be useful for analyzing not only bone thickness but also other important parameters. If more CT data are accumulated in future studies, they could be used in stud- ies to identify numerical associations between head size parameters and to develop predictive factors for specific items (e.g., temporal bone thickness). Moreover, when used together with the Size Korea data, our CT data will aid the development of specific bone conduction headsets for Koreans.

\section{Acknowledgments}

This work was supported by the Technology Innovation Program (or Industrial Strategic Technology Development Program) (20001819) funded By the Ministry of Trade, Industry \& Energy (MOTIE, Korea) and by Basic Science research program through the national research foundation of Korea (NRF) funded by the Ministry of Education, Science and Technology (NRF-2019K1A3A1A47000527).

\section{Conflicts of interest}

The authors have no financial conflicts of interest.

\section{Author Contributions}

Conceptualization: Soo Won Kim, Ji Young Kim, and Young Joon Seo. Data curation: Cheol Hyo Ku, Soo Won Kim, Ji Young Kim, Seung Won Paik, Hui Joon Yang, and Ji Hyeon Lee. Formal analysis: Cheol Hyo Ku, Soo Won Kim, Ji Young Kim, and Ji Hyeon Lee. Methodology: Young Joon Seo. Project administration: Young Joon Seo. Resources: Young Joon Seo. Software: Cheol Hyo Ku, Seung Won Paik, and Hui Joon Yang. Supervision: Young Joon Seo. Validation: Cheol Hyo Ku and Young Joon Seo. Visualization: Cheol Hyo Ku, Seung Won Paik, Hui Joon Yang, and Ji Hyeon Lee. Writing — original draft: Cheol Hyo Ku. Writing—review \& editing: Cheol Hyo Ku and Young Joon Seo.

\section{ORCID iDs}

Cheol Hyo $\mathrm{Ku}$

Soo Won Kim

Ji Young Kim

Seung Won Paik

Hui Joon Yang

Ji Hyeon Lee

Young Joon Seo https://orcid.org/0000-0002-0841-6030 https://orcid.org/0000-0002-4193-725X https://orcid.org/0000-0001-7905-8296 https://orcid.org/0000-0002-9680-8615 https://orcid.org/0000-0003-4735-1652 https://orcid.org/0000-0003-0896-9062 https://orcid.org/0000-0003-1169-0441

\section{REFERENCES}

1) Håkansson B, Tjellström A, Rosenhall U, Carlsson P. The bone-anchored hearing aid. Principal design and a psychoacoustical evaluation. Acta Otolaryngol 1985;100:229-39.

2) Kong TH, Kwak C, Han W, Seo YJ. Evaluation of wireless Bluetooth devices to improve recognition of speech and sentences when using a mobile phone in bone conduction device recipients. Eur Arch Otorhinolaryngol 2019 Jun 19 [Epub]. https://doi.org/10.1007/s00405019-05516-3.

3) Wolfe J, Morais Duke M, Schafer E, Cire G, Menapace C, O'Neill L. Evaluation of a wireless audio streaming accessory to improve mobile telephone performance of cochlear implant users. Int J Audiol 2016;55:75-82.

4) Dimitriadis PA, Hind D, Wright K, Proctor V, Greenwood L, Carrick $\mathrm{S}$, et al. Single-center experience of over a hundred implantations of a transcutaneous bone conduction device. Otol Neurotol 2017; 38:1301-7.

5) Lee HJ, Park SJ. Comparison of Korean and Japanese head and face anthropometric characteristics. Hum Biol 2008;80:313-30.

6) Hodgetts WE, Scollie SD, Swain R. Effects of applied contact force 
and volume control setting on output force levels of the BAHA Softband. Int J Audiol 2006;45:301-8.

7) Ball R, Shu C, Xi P, Rioux M, Luximon Y, Molenbroek J. A comparison between Chinese and Caucasian head shapes. Appl Ergon 2010;41:832-9.

8) Lindley AA, Benson JE, Grimes C, Cole TM 3rd, Herman AA. The relationship in neonates between clinically measured head circumference and brain volume estimated from head CT-scans. Early Hum Dev 1999;56:17-29.

9) Mudry A, Tjellström A. Historical background of bone conduction hearing devices and bone conduction hearing aids. Adv Otorhinolaryngol 2011;71:1-9.

10) Zhang L, Gao N, Yin Y, Yang L, Xie Y, Chen Y, et al. Bone conduction hearing in congenital aural atresia. Eur Arch Otorhinolaryngol 2016;273:1697-703.

11) Hougaard DD, Boldsen SK, Jensen AM, Hansen S, Thomassen PC. A multicenter study on objective and subjective benefits with a transcutaneous bone-anchored hearing aid device: first Nordic results. Eur Arch Otorhinolaryngol 2017;274:3011-9.

12) Gawliczek T, Wimmer W, Munzinger F, Caversaccio M, Kompis M. Speech understanding and sound localization with a new nonimplant- able wearing option for Baha. Biomed Res Int 2018;2018:5264124.

13) Verstraeten N, Zarowski AJ, Somers T, Riff D, Offeciers EF. Comparison of the audiologic results obtained with the bone-anchored hearing aid attached to the headband, the testband, and to the "snap" abutment. Otol Neurotol 2009;30:70-5.

14) Kim G, Ju HM, Lee SH, Kim HS, Kwon JA, Seo YJ. Efficacy of boneanchored hearing aids in single-sided deafness: a systematic review. Otol Neurotol 2017;38:473-83.

15) Seo YJ, Kwak C, Kim S, Park YA, Park KH, Han W. Update on boneconduction auditory brainstem responses: a review. J Audiol Otol 2018;22:53-8.

16) Reinfeldt $\mathrm{S}$, Håkansson $\mathrm{B}$, Taghavi $\mathrm{H}$, Eeg-Olofsson $\mathrm{M}$. New developments in bone-conduction hearing implants: a review. Med Devices (Auckl) 2015;8:79-93.

17) Baker AR, Fanelli DG, Kanekar S, Isildak H. A retrospective review of temporal bone imaging with respect to bone-anchored hearing aid placement. Otol Neurotol 2017;38:86-8.

18) Jang DS, Shin DH, Han W, Kong TH, Seo YJ. Baha attract implantation using a small incision: initial report of surgical technique and surveillance. Clin Exp Otorhinolaryngol 2019 Jul 6 [Epub]. https:// doi.org/10.21053/ceo.2019.00381. 